\title{
Conformal Regularization of the Kepler Problem
}

\author{
Bruno Cordani
}

Dipartimento di Matematica dell'Università, via Saldini 50, I-20133 Milano, Italy

\begin{abstract}
The manifold $M$ of null rays through the origin of $\mathbb{R}^{2, n+1}$ is diffeomorphic to $S^{1} \times S^{n}$, and it is a homogeneous space of $\operatorname{SO}(2, n+1)$. This group therefore acts on $T^{*} M$, which we show to be the "generating manifold" of the extended phase space of the regularized Kepler Problem. A local canonical chart in $T^{*} M$ is found such that the restriction to the subbundle of the null nonvanishing covectors is given by $p_{0}+H(q, p)=0$, where $H(q, p)$ is the Hamiltonian of the Kepler Problem. By means of this construction, we get some results that clarify and complete the previous approaches to the problem.
\end{abstract}

\section{Introduction}

By the Kepler Problem (KP) we mean the Hamiltonian system on the phase space $T^{*}\left(\mathbb{R}^{n}-0\right)$, with the Hamiltonian

$$
H=\frac{1}{2} p^{2}-\frac{1}{q}
$$

$q_{k}$ and $p_{k}$ being canonical coordinates, $p^{2}=\sum_{k} p_{k}^{2}$ and $q=\left(\sum_{k} q_{k}^{2}\right)^{1 / 2}$

Let us recall some well known facts:

i) As every particle in a spherically symmetric field, the KP is completely integrable. Besides the obvious integrals of energy and angular momentum, one has conservation of the Lenz-Laplace vector

$$
\left(\frac{\varepsilon}{2 H}\right)^{1 / 2}\left(p^{2} q_{k}-\frac{q_{k}}{q}-\langle q, p\rangle p_{k}\right),
$$

where $(E=$ numerical value of $H)$ :

$$
\begin{array}{ll}
\varepsilon=\operatorname{sgn} E & (E \neq 0), \\
\varepsilon=0 & (E=0) .
\end{array}
$$

For $E=0$ we define $(\varepsilon / 2 H)^{1 / 2}$ to be the modulus of the angular momentum. Under Poisson brackets, angular momentum and Lenz-Laplace vector yield the Lie algebra of $\mathrm{SO}(n+1), \mathrm{SO}(n, 1)$ or $\mathrm{SO}(n) \otimes_{S} \mathbb{R}^{n}$ (semidirect product) for negative, positive or null energy. These groups are the maximal invariance groups. Fock [1] in 
the quantum 3-dimensional case and Moser [2] in the classical $n$-dimensional case gave a geometrical picture of this dynamical symmetry (for $E<0$ ) through the stereographic projection of the sphere $S^{n}$ in the momentum space. In this way one obtains at the same time the regularization of the KP. In fact:

ii) The KP is not regular, i.e. the vector field generated by $H$ in $T^{*}\left(\mathbb{R}^{n}-0\right)$ is not complete since, in the collision orbits, the particle gets to the attractive center with infinite velocity in a finite time. Following Pham Mau Quan [3] we define "regularization" in this way: given a smooth manifold $W$ and a non-complete vector field $X$ on $W$, find a smooth manifold $\tilde{W}$, a complete vector field $\tilde{X}$ on $\tilde{W}$ and an embedding $\mu: W \mapsto \tilde{W}$ such that $\mu(W)$ is a set open and dense in $\tilde{W}$ and the orbits of $X$ are embedded in those of $\tilde{X}$. The regularization of the 3-dimensional KP may be also obtained by the Kustaanheimo-Stiefel-transformation [4][5]. Kummer [6] proved the equivalence of the two methods for $E \neq 0$. In both these regularization procedures, time is replaced by a new parameter $\alpha$ such that

$$
\frac{d t}{d \alpha}=q .
$$

It follows that:

iii) For any fixed value of $E<0$ the phase space of the regularized KP is diffeomorphic to the unit $T^{*} S^{n}$. Notice that $T^{+} S^{n}$ (i.e. $T^{*} S^{n}$ with the zero section removed) is an orbit in the coadjoint representation of $\operatorname{SO}(2, n+1)$, which is locally isomorphic to the conformal group of $\mathbb{R}^{1, n}[7-9]$. This dynamical group has been defined by Bacry [10] and Györgyi [11], who also introduced the so called BacryGyörgyi variables, to be used alternatively to Fock variables (see [9] for the definitions).

In this paper we proceed as follows. The manifold $M$ of null unoriented rays through the origin of $\mathbb{R}^{2, n+1}$ is diffeomorphic to $S^{1} \times S^{n}$, which is in turn a homogeneous space of $G=\mathrm{SO}(2, n+1)$. This group acts therefore on $T^{*} M$. As we shall see later, $T^{*} M$ can be identified with the "generating manifold" of the extended phase space of the regularized KP with any energy. The action of $G$ on $T^{*} M$ is not transitive, so we restrict to consider the $(2 n+1)$-dimensional subbundle $N$ of $T^{*} M$ given by null non-vanishing covectors: it results that $T^{+} S^{n}=N / S^{1}$. The main point is the following: it is possible to find three local canonical charts in $T^{*} M$ (one for every value of $\varepsilon$ ) such that $N$ is locally given by the equation

$$
p_{0}+H(q, p)=0 \text {, }
$$

where $H(q, p)$ is the Hamiltonian (1.1) of the KP or the Hamiltonian with repulsive potential.

As is well known (see Theorem 2.6 below), by considering (1.5) as a constraint in $T^{*}\left(\mathbb{R}^{n}-0\right) \times T^{*} \mathbb{R}$ one obtains the Hamilton equations

$$
\frac{d q_{k}}{d q_{0}}=\frac{\partial H}{\partial p_{k}}, \quad \frac{d p_{k}}{d q_{0}}=-\frac{\partial H}{\partial q_{k}} .
$$

By means of this construction, we get some results that clarify and complete the previous approaches to the KP: 
a) the introduction of the regularization parameter $\alpha$ in Eq. (1.4) is not postulated, but is a consequence of our approach;

b) the definition of Fock and Bacry-Györgyi variables is extended to the case $E=0$, and their relations are clarified;

c) the case of repulsive potential is automatically included;

d) the equivalence between Fock-Moser and $\mathrm{KS}$ regularization, which is basically due to the homomorphism $\mathrm{SO}(2,4) \simeq \mathrm{SU}(2,2)$, is here straightforwardly proved for any value of $E$;

e) as suggested in [12], the present construction can be generalized by considering the simple Lie groups whose maximal compact subgroup contains U(1) [13, Chap. VIII], and studying their action on the Bergman-Silov boundary;

f) as we shall show in a forthcoming paper, the geometric quantization (in the sense of Kostant and Souriau) of the Kepler manifold $T^{+} S^{n}$ can be naturally obtained. Notice, for instance, that $N$ is already the prequantum bundle.

As for the notation, the range of the indices is

$$
\begin{aligned}
A, B, C & =-1,0 \ldots n+1, \\
\mu, v, \rho & =0 \ldots n, \\
\alpha, \beta, \gamma & =1 \ldots n+1, \\
i, j, k & =1 \ldots n, \\
a, b, c & =2 \ldots n .
\end{aligned}
$$

\section{The "Generating Manifold"}

Let $\eta_{A B}=\operatorname{diag}(--+\cdots+)$ be the metric tensor of $\mathbb{R}^{2, n+1}$ and $m_{A B}=-m_{B A}$ a basis of the Lie algebra $\mathscr{G}$ of $G=\mathrm{SO}(2, n+1)$. Then

$$
\left[m_{A B}, m_{A C}\right]=\eta_{A A} m_{B C},
$$

or zero if all indices are different. It is convenient to introduce special symbols for the elements of the basis, namely:

$$
\begin{aligned}
m_{\mu \nu} & =J_{\mu \nu}, \\
m_{\mu n+1} & =A_{\mu}, \\
m_{-1 \mu} & =B_{\mu}, \\
m_{-1 n+1} & =D ;
\end{aligned}
$$

or alternatively:

$$
\begin{aligned}
& P_{\mu}=A_{\mu}+B_{\mu}, \\
& C_{\mu}=A_{\mu}-B_{\mu} .
\end{aligned}
$$

We now recall some well known facts $[14,15]$. Since the action of $G$ on $\mathbb{R}^{2, n+1}$ is linear, it induces an action on the projective manifold of (unoriented) rays through the origin. Moreover $G$ sends the null cone into itself and acts transitively on the manifold $M$ of null rays. This manifold is diffeomorphic to $S^{1} \times S^{n}$ and is endowed with a class of pseudoriemannian metrics $g_{\gamma}$ obtained by restriction of the $\mathrm{SO}(2, n+1)$ invariant metric $\eta$ on any section $\gamma$ of the null cone. The action of $G$ on $M$ is conformal; the metrics $g_{\gamma}$ being conformaly flat, with signature $(-+\cdots+)$, 
the Lie algebra $\mathscr{G}$ of $G$ is isomorphic to the Lie algebra of conformal vector fields on Minkowski space $\mathbb{R}^{1, n}$. So we can identify the generators in $(2.2 a b)$ as follows: $J_{\mu \nu}=$ Lorentz group, $D=$ dilation, $P_{\mu}=$ translations, $C_{\mu}=$ conformal translations. Let $H$ be the (closed) subgroup of $G$ with Lie algebra $\mathscr{H}=\left\{J_{\mu \nu}, C_{\mu}, D\right\}:$ it is the isotropy group of the origin in $\mathbb{R}^{1, n}$. Since $M=G / H$, we can identify $M$ with the "conformal compactification" of $\mathbb{R}^{1, n}$. In other words, one can obtain $M$ by adding to $\mathbb{R}^{1, n}$ a null cone at infinity. ${ }^{1}$

Let us now consider the symplectic action of $G$ on $T^{*}(G / H)$. This action not being transitive, we decompose $T^{*}(G / H)$ in orbits of $G$. They are symplectic manifolds on which the group action is transitive, and so they may be identified (Kostani-Souriau Theorem, [16, p. 180] with (covering spaces of) orbits of $G$ in $\mathscr{G}^{*}$. To get this identification, it is useful the following theorem due to Wolf [17]:

Theorem 2.1. Let $G$ be a Lie group with Lie algebra $\mathscr{G}, f \in \mathscr{G}^{*}, G_{f}$ the isotropy subgroup of $f\left(\right.$ i.e. $\left.G_{f} \cdot f=f\right)$ and $\mathscr{G}_{f}$ the corresponding Lie algebra. Consider a closed subgroup $H \subset G$ with Lie algebra $\mathscr{H}$ such that: a) $\left.\operatorname{dim} \mathscr{H}=\frac{1}{2}\left(\operatorname{dim} \mathscr{G}+\operatorname{dim} \mathscr{G}_{f}\right) ; \mathrm{b}\right)$ $\langle f, \mathscr{H}\rangle=0 ; \mathrm{c}) \mathscr{G}_{f} \subset \mathscr{H}$. Then $O_{f}=G \cdot f$ is equivariantly diffeomorphic to an open $G$ orbit in $T^{*}(G / H)$.

If, as in the present case, $G$ is semisimple, by means of the Cartan-Killing form $B: \mathscr{G} \times \mathscr{G} \mapsto \mathbb{R}$ we may identify $\mathscr{G}$ and $\mathscr{G}^{*}$. So, for $m \in \mathscr{G}$, we define $m^{*} \in \mathscr{G}^{*}$ by $\left\langle m^{*}, n\right\rangle=B(m, n), \forall n \in \mathscr{G}$. Therefore $\mathscr{G}_{m^{*}}=\{n \in \mathscr{G}:[m, n]=0\}$. The basis $(2.2 a)$ is (pseudo)-orthonormal for $B$ and so $B\left(P_{\mu}, C_{v}\right)=2 \eta_{\mu \nu}, B(P, P)=B(C, C)=0$.

Proposition 2.2. If $f_{-}=C_{0}^{*}$ and $f_{+}=C_{1}^{*}$, then $\mathcal{O}_{f_{-}}\left(\mathcal{O}_{f_{+}}\right)$are the submanifolds of $T^{*}(G / H)$ given by timelike (spacelike) covectors.

Proof. $\mathscr{G}_{f_{-}}=\left\{C_{\mu}, J_{h k}\right\}$ and $\mathscr{G}_{f_{+}}=\left\{C_{\mu}, J_{a 0}, J_{a b}\right\}$, then $\mathscr{H}=\left\{J_{\mu \nu}, C_{\mu}, D\right\}$ satisfies the hypotheses of Theorem 2.1. Moreover $G / H$ has tangent space $\mathscr{G} / \mathscr{H}$, hence cotangent space $\mathscr{H}^{\perp}=\left\{x \in \mathscr{G}^{*}:\langle x, \mathscr{H}\rangle=0\right\}$, and so $\mathscr{H}^{\perp}=\left\{C_{\mu}^{*}\right\}$. Remembering the signature of $g_{\gamma}$ and that the action of $G$ on $G / H$ is conformal, the proposition is obtained.

Proposition 2.3. The symplectic $G$ invariant form induced in $\mathcal{O}_{f_{\mp}}$ by the canonical form of $T^{*}(G / H)$ through the equivariant diffeomorphism of Theorem 2.1, coincides with the Kirillov form.

Proof. We remember that the Kirillov 2-form $\omega$, (that makes every orbit of a group in the coadjoint representation a symplectic manifold) is defined as

$$
\omega_{f}(u, v)=\langle f,[u, v]\rangle,
$$

where $u, v \in$ to the Lie algebra of the group, and $f \in$ to the dual. The cotangent space to $\mathcal{O}_{f}$ in $f$ is spanned by $\mathscr{G} / \mathscr{G}_{f}$ : for, e.g., $f_{-}$we have $\mathscr{G}_{/} / \mathscr{G}_{f-}=\left\{P_{\mu} ; D, J_{0 k}\right\}$, where $\left\{P_{\mu}\right\}$ span the tangent space to $G / H$. A direct computation of (2.3) proves the proposition. Analogously for $f_{+}$.

The two orbits $\mathcal{O}_{f_{\mp}}$ are $2(n+1)$-dimensional. We now come to the $2 n$ dimensional orbit considered by Onofri [8] and called "Kepler manifold."

1 The relation of the conformal compactification with the regularization of the KP has been noted first by Kummer [6] 
Proposition 2.4. Let $f_{0}=f_{+}-f_{-}$: then $\mathcal{O}_{f_{0}}$ is symplectomorphic to $T^{+} S^{n}$, endowed with the canonical symplectic form.

Proof. Let $G^{\prime}=\mathrm{SO}(1, n+1)$. As a subgroup of $G, G^{\prime}$ has the Lie algebra $\mathscr{G}^{\prime}=$ $\left\{J_{h k}, P_{k}, C_{k}, D\right\}$. Consider the subgroup $H^{\prime}$ of $G^{\prime}$ generated by $\mathscr{H}^{\prime}=\left\{J_{h k}, C_{k}, D\right\}$. The manifold $M^{\prime}=G^{\prime} / H^{\prime}$ can be identified with the projective manifold of the rays of the null cone in $\mathbb{R}^{1, n+1}$, and so $M^{\prime}=S^{n}$. Being $f_{+}=C_{1}^{*}$, then $\mathscr{G}_{f_{+}}^{\prime}=\left\{C_{k}, J_{a b}\right\}$. From Theorem 2.1 we obtain: $\mathcal{O}_{f_{+}}^{\prime}=T^{+} S^{n}$, where $\mathcal{O}_{f_{+}}^{\prime}=G^{\prime} \cdot f_{+}$. Let $\mathscr{P}$ be the orthogonal complement of $\mathscr{G}^{\prime}$ in $\mathscr{G}$, i.e. $B\left(\mathscr{P}, \mathscr{G}^{\prime}\right)=0$ and $\mathscr{G}=\mathscr{G}^{\prime} \oplus \mathscr{P}$. Being $G^{\prime} \cdot \mathscr{P}^{*}=\mathscr{P}^{*}$ and $f_{+} \in \mathscr{G}^{\prime *}, f_{-} \in \mathscr{P}^{*}$, we have that $G^{\prime} \cdot f_{0}$ is identifiable, through projection $\mathscr{G}^{*} \mapsto \mathscr{G}^{\prime *}$, with $\mathcal{O}_{f_{+}}^{\prime}$. The orbit $G \cdot f_{0}$ contains $G^{\prime} \cdot f_{0}$, but a dimension count shows that they must coincide: therefore we obtain the proposition. Computing $\mathscr{G}_{f_{0}}$ hence the cotangent space to $\mathcal{O}_{f_{0}}$, shows that the Kirillov form coincides with the canonical symplectic form of $T^{+} S^{n}$.

Analogously, we could prove Proposition 2.4 for $f_{0}=f_{+}+f_{-}$. Thus summarizing, we have

$$
N / S^{1}=\mathcal{O}_{f_{0}}\left(\bmod \mathbb{Z}_{2}\right),
$$

where $N$ is the submanifold of null non-vanishing covectors in $T^{*} M$.

Proposition 2.5. Identify $\mathscr{G}^{*}$ with $\wedge^{2} \mathbb{R}^{2, n+1}$, then $T^{*} M-$ (zero-section) is diffeomorphic to the manifold of the simple null non-vanishing bivectors, i.e. the bivectors of the type $Y \wedge X$, where $X, Y \in \mathbb{R}^{2, n+1}$ and $\eta(X, X)=0, \eta(X, Y)=0$.

Proof. It is sufficient to verify that: a) $f_{\text {- }}$ as simple bivector is generated by $X=$ $(-10 \ldots 01)$ and $Y=(010 \ldots 0) ;$ b) $f_{+}$by $X=$ idem and $Y=(0010 \ldots 0)$; c) $f_{0}$ by $X=$ idem and $Y=(0110 \ldots 0)$.

The following fact is crucial for our concern: the reduction $T^{*} M \mapsto T^{+} M^{\prime}$ above described, may be interpreted as the reduction of the extended phase space of a mechanical system to the phase space. More exactly, we have the following classical theorem (see e.g. [18]):

Theorem 2.6. Let $\mathscr{K}: T^{*} Q \mapsto \mathbb{R}$ be a "time" independent Hamiltonian, and $\left(x_{0}, x_{k}, y_{0}, y_{k}\right)$ the canonical coordinates of $T^{*} Q$ ( $Q$ is any differentiable manifold $)$. Therefore $\mathscr{K}$ equals some constant $h$, and we may write, at least locally,

$$
y_{0}+K\left(x_{0}, x_{k}, y_{k}\right)=0 \text {. }
$$

Let us project the trajectories generated by $\mathscr{K}$ and belonging to the hypersurface (2.5) onto the hyperplane $y_{0}=0$ : they are the solution of the hamiltonian system

$$
\frac{d x_{k}}{d x_{0}}=\frac{\partial K}{\partial y_{k}}, \quad \frac{d y_{k}}{d x_{0}}=-\frac{\partial K}{\partial x_{k}} .
$$

In our case $Q=M$ and $\mathscr{K}=g_{\gamma}(y, y)$ with $h=0$. Notice that $g_{\gamma}$ is conformally flat $\forall \gamma$, so we can choose local coordinates $\left(x_{0}, x_{k}\right)$ on $M$ such that $g_{\gamma}$ is diagonal with $x_{0}$ timelike. Then $x_{0}$ is a local coordinate on the manifold of null rays in $\mathbb{R}^{2,1} \subset \mathbb{R}^{2, n+1}$. Apply Theorem 2.6: the reduced phase space is $T^{+} M^{\prime}=\mathcal{O}_{f_{0}}$ and $K=$ $\mp\left(g_{\gamma^{\prime}}\left(y^{\prime}, y^{\prime}\right)\right)^{1 / 2}$. 
Three choices of $\gamma$ are relevant for the KP, i.e. those obtained intersecting the null cone with:

i) a sphere with center in the vertex of the cone, thus $\gamma$ is defined on $M=S^{1} \times S^{n}$ and $\gamma^{\prime}$ on $M^{\prime}=S^{n}$ with the usual metric induced by the immersion of $S^{n}$ in $\mathbb{R}^{n+1}$ (more exactly, being $x_{0}$ a coordinate of the "time" type, we consider the universal covering $\widetilde{G}$ instead of $G$, and so $\tilde{M}=\mathbb{R} \times S^{n}$ instead of $M$ );

ii) a hyperboloid with same center; $\gamma$ is defined on $M-\mathbb{Z}_{2} \times C_{\infty}=H^{1} \times H^{n}$, where $C_{\infty}$ is the null cone at infinity in $M$ and the $H$ s are hyperboloids (the metric in $H^{n}$ is induced by the immersion in $\left.\mathbb{R}^{1, n}\right) ; \gamma^{\prime}$ is defined on $M^{\prime}-($ two points at $\infty)=H^{n}$;

iii) a hyperplane parallel to a ray of the null cone; $\gamma$ is defined on $M-C_{\infty}=P^{1, n}$ (hyperbolic paraboloid) and $\gamma^{\prime}$ on $M^{\prime}$ - (one point at $\left.\infty\right)=P^{n}$; the two metric are flat.

The Hamiltonian $K$ is the Hamiltonian of the unit geodesic flows on i) $S^{n}$, ii) $H^{n}$, iii) $P^{n}$ and the invariance groups (i.e. the isometry groups of $\left.g_{\gamma}\right)$ are i) $\mathrm{SO}(n+1)$, ii) $\mathrm{SO}(n, 1)$, iii) $\mathrm{SO}(n) \otimes_{S} \mathbb{R}^{n}$.

The main point of the present work is the following

Theorem 2.7. The extended phase space of the regularized $\mathrm{KP}$ (for negative, positive and null E) is symplectomorphic to the open submanifolds of $T^{*} M$ given by the domain of the sections $\gamma$ defined in i) ii) iii) (in this sense $T^{*} M$ is the "generating manifold"). The Hamiltonian of the KP is a function of $K$ and so has the same symmetry groups.

\section{Regularization of the KP}

In this section we prove the theorem above and the points a), b) and c) of Sect. 1. To this end we construct the moment map $T^{*} M \mapsto \mathscr{G}^{*}$, in the three cases, using the following construction suggested by Proposition 2.5 . Since $\gamma: M \mapsto \mathbb{R}^{2, n+1}$ is a section of the null cone, we can locally represent it by functions

$$
X^{A}=\Gamma^{A}\left(x^{\mu}\right)
$$

satisfying the null cone equation,

$$
\eta(\Gamma, \Gamma)=0 .
$$

The metric induced on the domain of $\gamma$ by $\eta$ is given by $g_{\gamma \mu \nu}=\psi_{\mu}^{A} \eta_{A B} \psi_{v}^{B}$, where $\psi_{\mu}^{A}=\partial \Gamma^{A} / \partial x^{\mu}$. Let $Y_{A}$ and $y_{\mu}$ be the components of a covector respectively of $\mathbb{R}^{2, n+1}$ and $M$. Let $T^{*} \gamma: T^{*} M \mapsto T^{*} \mathbb{R}^{2, n+1}$ be the cotangent map, i.e. the map locally given by (3.1) and by $Y_{A}=\Pi_{A}\left(x^{\mu}, y_{v}\right)$, where

$$
\Pi_{A}=\eta_{A B} \psi_{\mu}^{B} g_{\gamma}^{\mu v} y_{v}
$$

It is easy to check that

$$
\begin{aligned}
\Pi_{A} \Gamma^{A} & =0, \\
\Pi_{A} d \Gamma^{A} & =y_{\mu} d x^{\mu} .
\end{aligned}
$$

If $f$ and $g$ are differentiable mappings: $T^{*} \mathbb{R}^{2, n+1} \mapsto \mathbb{R}$, from (3.5) we have: $\{f, g\}$. $T^{*} \gamma=\left\{f \cdot T^{*} \gamma, g \cdot T^{*} \gamma\right\}$, where $\{\cdot, \cdot\}$ are the Poisson brackets. If $j: T^{*} \mathbb{R}^{2, n+1} \mapsto \mathscr{G} *$ is the moment map

$$
m_{A B}=Y_{A} X_{B}-Y_{B} X_{A},
$$

then $J=j \cdot T^{*} \gamma: T^{*} M \mapsto \mathscr{G}^{*}$ is a moment map as well. 
Explicitly we have the following three cases.

i) $T^{*} \gamma$ is given by

$$
\begin{aligned}
X^{-1} & =\cos x^{0}, \\
X^{0} & =\sin x^{0}, \\
X^{k} & =\frac{2 x^{k}}{x^{2}+1}, \\
X^{n+1} & =\frac{x^{2}-1}{x^{2}+1},
\end{aligned}
$$

and by

$$
\begin{aligned}
Y_{-1} & =-y_{0} \sin x^{0}, \\
Y_{0} & =y_{0} \cos x^{0}, \\
Y_{k} & =\frac{1}{2}\left(x^{2}+1\right) y_{k}-\langle x, y\rangle x_{k}, \\
Y_{n+1} & =\langle x, y\rangle .
\end{aligned}
$$

Notice that $x^{0}$ do not parametrize $S^{1}$ but rather its covering space $\simeq \mathbb{R}$. The functions $\Gamma^{\alpha}$ are obtained through a stereographic projection of $S^{n}$ onto $\mathbb{R}^{n}$. The metric $g_{\gamma}$ is

$$
\|y\|^{2}=-y_{0}^{2}+\left[\frac{1}{2} y\left(x^{2}+1\right)\right]^{2},
$$

where $y=\left(\sum y_{k} y_{k}\right)^{1 / 2}$ and $x^{2}=\sum x^{k} x^{k}$.

ii) $T^{*} \gamma$ is given by

$$
\begin{aligned}
X^{-1} & =\frac{x^{2}+1}{x^{2}-1}, \\
X^{0} & =\operatorname{Sinh} x^{0}, \\
X^{k} & =\frac{2 x^{k}}{x^{2}-1}, \\
X^{n+1} & =\operatorname{Cosh} x^{0},
\end{aligned}
$$

and by

$$
\begin{aligned}
Y_{-1} & =\langle x, y\rangle \\
Y_{0} & =y_{0} \operatorname{Cosh} x^{0} \\
Y_{k} & =\frac{1}{2}\left(x^{2}-1\right) y_{k}-\langle x, y\rangle x_{k}, \\
Y_{n+1} & =-y_{0} \operatorname{Sinh} x^{0} .
\end{aligned}
$$

The functions $\Gamma^{-1}$ and $\Gamma^{k}$ are obtained through a stereographic projection of one sheet of $H^{n}$ into $\mathbb{R}^{n}$ (i.e. onto the $n$-dimensional Poincaré disk) and a following inversion with respect to the origin, so that we have $x^{2}>1$. The metric $g_{\gamma}$ is

$$
\|y\|^{2}=-y_{0}^{2}+\left[\frac{1}{2} y\left(x^{2}-1\right)\right]^{2} .
$$


iii) $T^{*} \gamma$ is given by

and by

$$
\begin{aligned}
X^{-1} & =1+\frac{1}{x^{2}}-\frac{\left(x^{0}\right)^{2}}{4}, \\
X^{0} & =x^{0} \\
X^{k} & =\frac{2 x^{k}}{x^{2}} \\
X^{n+1} & =1-\frac{1}{x^{2}}+\frac{\left(x^{0}\right)^{2}}{4},
\end{aligned}
$$

$$
\begin{aligned}
Y_{-1} & =\frac{1}{2}\left[\langle x, y\rangle-x^{0} y_{0}\right], \\
Y_{0} & =y_{0}, \\
Y_{k} & =\frac{1}{2} x^{2} y_{k}-\langle x, y\rangle x_{k}, \\
Y_{n+1} & =\frac{1}{2}\left[\langle x, y\rangle-x^{0} y_{0}\right] .
\end{aligned}
$$

The mapping is obtained through a projection of $P^{1, n}$ onto $\mathbb{R}^{1, n}$ and a following inversion with respect to the origin in $\mathbb{R}^{n}$, so that we have $x^{2} \neq 0$. The metric $g_{\gamma}$ is given by

$$
\|y\|^{2}=-y_{0}^{2}+\left[\frac{1}{2} y x^{2}\right]^{2} \text {. }
$$

We stress the fact that, owing to stereographic projection in i) plus inversion in ii) and iii), we are missing one point in $S^{n}, H^{n}$ and $P^{n}$ : restoring this point corresponds just to regularization of the KP.

From Theorem 2.6 we obtain that the Hamiltonian $K$ is given in the three cases by

$$
K=\frac{1}{2} y\left(x^{2}-\varepsilon\right),
$$

where $\varepsilon$ is defined in (1.3). Let us reduce the three moment maps, i.e., in accordance with (2.4), put $\mathscr{K}=0$ and $x^{0}=0$, and consider the unregularized problem. All the three moment maps $T^{+} \mathbb{R}^{n} \mapsto \mathscr{G}^{*}$ now become (see [19, p. 276] for the precise definition of $\hat{J}, \hat{A}$ etc.)

and

$$
\begin{aligned}
\hat{J}_{h k} & =y_{h} x_{k}-y_{k} x_{h}, \\
\hat{J}_{0 k} & =-y x_{k}, \\
\hat{A}_{0} & =-\frac{1}{2} y\left(x^{2}-1\right), \\
\hat{A}_{k} & =\frac{1}{2}\left(x^{2}-1\right) y_{k}-\langle x, y\rangle x_{k}, \\
\hat{B}_{0} & =-\frac{1}{2} y\left(x^{2}+1\right), \\
\hat{B}_{k} & =\frac{1}{2}\left(x^{2}+1\right) y_{k}-\langle x, y\rangle x_{k}, \\
\hat{D} & =\langle x, y\rangle
\end{aligned}
$$

$$
\begin{aligned}
& \hat{P}_{0}=-y x^{2}, \\
& \hat{P}_{k}=x^{2} y_{k}-2\langle x, y\rangle x_{k}, \\
& \hat{C}_{0}=y, \\
& \hat{C}_{k}=-y_{k} .
\end{aligned}
$$


The Hamiltonian $K$ equals in the three cases (modulo an uninteresting sign): i) $\hat{B}_{0}$, ii) $\hat{A}_{0}$, iii) $\hat{P}_{0} / 2$ and thus have as symmetry groups the isotropy subgroups of these generators, i.e.: i) $\mathrm{SO}(n+1)$, ii) $\mathrm{SO}(1, n)$, iii) $\mathbb{R}^{n} \otimes_{S} \mathrm{SO}(n)$.

Let us return to the moment map $J$ and before reducing it, consider the canonical transformation $\mathscr{C}$

$$
\begin{aligned}
& q_{k}=y_{0} y_{k} \\
& p_{k}=-\frac{x_{k}}{y_{0}}, \\
& q_{0}=\frac{y_{0}^{3}}{\varepsilon}\left[x_{0}-\frac{\langle x, y\rangle}{y_{0}}\right], \\
& p_{0}=\frac{\varepsilon}{-2 y_{0}^{2}} .
\end{aligned}
$$

$(\mathscr{C}$ is not defined for $\varepsilon=0$. However, since $\varepsilon$ enters in the formulas below only through the expression $(\varepsilon / 2 H)^{1 / 2}$, which we defined in the limit case also, we can safely take the limit $\varepsilon=0$ in the final formulas.) $\mathscr{C}$ may be viewed as the composition of three canonical transformations: a) that given by exchanging coordinates and momenta; b) that given by ( $3.15 a b)$, equivalent to an "energy rescaling"; c) that given by $(3.15 d)$. Note that $(3.15 c)$ is forced by requiring canonicity. Now $\mathscr{K}=0$ reads as

$$
p_{0}+H(q, p)=0 \text {, }
$$

where $H(q, p)=p^{2} / 2 \mp q^{-1}$. Equation (3.15d) shows that $H$ is a function of $K$, so it has the same symmetry groups. Note that $x^{0}$ is basically the regularization parameter: in fact

$$
\frac{d q_{0}}{d x^{0}}=\left\{K, q_{0}\right\}+\frac{\partial q_{0}}{\partial x_{0}}
$$

and setting $\alpha=(\varepsilon / 2 H)^{1 / 2} x^{0}$, we get (1.4).

Let us consider the restriction to $N$ of the moment maps $J \cdot \mathscr{C}^{-1}: T^{*}\left(\mathbb{R}^{n}-0\right) \times$ $T^{*} \mathbb{R} \mapsto \mathscr{G}^{*}$. We have

i) $\widehat{B}_{0}=(-2 H)^{-1 / 2} ; \hat{J}_{h k}=$ angular momentum; $\hat{A}_{k}=$ Lenz-Laplace vector; $\widehat{B}_{k}, \hat{D}$ and $\hat{J}_{0 k}, \hat{A}_{0}=$ Fock variables (for $x^{0}=0$ ) or Bacry-Györgyi variables (for $q_{0}=0$ ).

ii) $\hat{A}_{0}=(2 H)^{-1 / 2} ; \hat{J}_{h k}=$ angular momentum; $\hat{B}_{k}=$ Lenz-Laplace vector; $\hat{D}, \hat{A}_{k}$ and $-\widehat{B}_{0}, \hat{J}_{0 k}=$ Fock variables (for $x^{0}=0$ ) or Bacry-Györgyi variables (for $q_{0}=0$ ).

iii) $\hat{P}_{0} / 2 \mapsto(2 H / \varepsilon)^{-1 / 2}$ (in the limit sense); $\hat{J}_{h k}=$ angular momentum; $\hat{P}_{k}=$ LenzLaplace vector; $\hat{C}_{\mu}$ and $\hat{D}, \hat{J}_{0 k}=$ Fock variables (for $x^{0}=0$ ) or Bacry-Györgyi variables (for $q_{0}=0$ ).

\section{KS-transformation}

As Kummer proved, the local isomorphism $\mathrm{SO}(4,2) \simeq \mathrm{SU}(2,2)$ yields the $\mathrm{KS}$ transformation for $E \neq 0$. We first recall some of the Kummer's results. Let $\mathscr{E}$ be a matrix representation of the $\mathrm{U}(2,2)$ invariant Hermitian form. We can choose a basis in $\mathbb{C}^{2,2}$ such that $\mathscr{E}$ has the form 


$$
\mathscr{E}=\left(\begin{array}{cc}
0 & \sigma_{0} \\
\sigma_{0} & 0
\end{array}\right),
$$

being $\sigma_{v}$ the Pauli matrices. Following Penrose we call twistors the elements of $\mathbb{C}^{2,2}$ on which $\mathrm{U}(2,2)$ acts in the fundamental representation, and null twistor space $T_{0}$ the set of elements $\phi \in \mathbb{C}^{2,2}$ such that

$$
\phi^{\dagger} \mathscr{E} \phi=0
$$

Identifying the null twistors up a phase, i.e. $\phi \approx \phi \exp (i \theta)$, we get that the quotient $T_{0} / \approx$ is a real 6-dimensional manifold. Let $z \in \mathbb{C}^{2}-0$ and $w \in \mathbb{C}^{2}$ be such that $\psi=\left(\begin{array}{l}z \\ w\end{array}\right) \in T_{0}$. It is easy to check that the matrices of the type

$$
i \psi \psi^{\dagger} \mathscr{E}=i\left(\begin{array}{cc}
z w^{\dagger} & z z^{\dagger} \\
w w^{\dagger} & w z^{\dagger}
\end{array}\right)
$$

describe a 6-dimensional orbit of $\operatorname{SU}(2,2)$ in $\operatorname{su}^{*}(2,2)$. This orbit is equipped with the symplectic form $\omega=d \Theta$, where

$$
\Theta=\frac{i}{2}\left(\psi^{\dagger} \mathscr{E} d \psi-d \psi^{\dagger} \mathscr{E} \psi\right)
$$

On the basis of this construction, then Kummer proves the equivalence between Fock-Moser and KS regularization.

As an application of the present approach we prove the same result. As a byproduct we get the KS-transformation in a way which is independent of the sign of $E$ and also covers the case $E=0$. Let $\Xi=\vec{x} \cdot \vec{\sigma}$ and $2 \Upsilon=y \sigma_{0}+\vec{y} \cdot \vec{\sigma}$. Being det $\Upsilon=0$, we can define $\Upsilon^{1 / 2}$ as an element of $\left(\mathbb{C}^{2}-0\right) / \approx$ such that $\Upsilon^{1 / 2} \bar{\Upsilon}^{+1 / 2}=\Upsilon$. Now

$$
\psi=\left(\begin{array}{c}
\Upsilon^{1 / 2} \\
i \Xi \Upsilon^{1 / 2}
\end{array}\right)
$$

provides a canonical system of coordinates for our orbit. In fact

$$
\Theta=\langle y, d x\rangle \text {. }
$$

The inverse of the bijective mapping (4.5) is the KS-transformation. To show it, immediately we have, from the mere definition, that

$$
r=z z^{\dagger}
$$

Moreover multiply from the right both sides of $-i w=\Xi z$ by $z^{\dagger}\left(z^{\dagger} z\right)^{-1}$ and take the imaginary part. We obtain

$$
\frac{1}{2} \frac{i}{z^{\dagger} z}\left(z w^{\dagger}-w z^{\dagger}\right)=\Xi+\sigma_{0} \frac{\langle x, y\rangle}{y} .
$$

Equations (4.7ab) are easily seen to be equivalent to the KS-transformation as given by Kummer [6].

The relation with the KP is seen by composing (4.3) with (4.5), which gives

$$
i \psi \psi^{\dagger} \mathscr{E}=i\left(\begin{array}{cc}
-i \Upsilon \Xi & \Upsilon \\
\Xi \Upsilon \Xi & i \Xi \Upsilon
\end{array}\right),
$$


and taking into account the isomorphism $\operatorname{su}^{*}(2,2)=\mathrm{so}^{*}(2,4)$. In this way we obtain the moment map (3.14ab), which is valid for any value of $E$.

\section{References}

1. Fock, V.: Theory of the hydrogen atom (in German). Z. Phys. 98, 145-154 (1935)

2. Moser, J.: Regularization of Kepler's problem and the averaging method on a manifold. Commun. Pure Appl. Math. 23, 609-636 (1970)

3. Pham Mau Quan. Riemannian regularization of singularities. Application to the Kepler problem. Proc. IUTAM-ISIMM Symp., Atti Accad. Sci. Torino 117, 341-348 (1983)

4. Kustaanheimo, P., Stiefel, E.: Perturbation theory of Kepler motion based on spinor regularization. J. Reine Angew. Math. 218, 204-219 (1965)

5. Stiefel, E., Scheifele, G.: Linear and regular celestial mechanics. Berlin, Heidelberg, New York: Springer 1971

6. Kummer, M.: On the regularization of the Kepler problem. Commun. Math. Phys. 84, 133-152 (1982)

7. Onofri, E., Pauri, M.: Dynamical quantization. J. Math. Phys. 13, 533-543 (1972)

8. Onofri, E.: Dynamical quantization of the Kepler manifold. J. Math. Phys. 17, 401-408 (1976)

9. Souriau, J. M.: Sur la varieté de Kepler. Symp. Math. 14, 343-360 (1974)

10. Bacry, H.: The de Sitter group $L_{4,1}$ and the bound states of hydrogen atom. Nuovo Cimento 41A, 222-234 (1966).

11. Györgyi, G.: Kepler's equation, Fock variables, Bacry generators and Dirac brackets. Nuovo Cimento 53A, 717-736 (1967)

12. Cordani, B.: Bergman-Šilov boundary and integrable Hamiltonian system (in Italian). Atti VII Congresso AIMETA-Univ. Trieste, Sez. 1, 175-182 (1984)

13. Helgason, S.: Differential geometry, Lie groups, and symmetric spaces, New York: Academic Press 1978

14. Penrose, R.: Relativistic symmetry groups, in: Group theory in non-linear problems Barut A.O. (ed.). Dordrecht: Reidel Publishing Company 1974

15. Sternberg, S.: On the influence of fields theories on our physical conception of geometry. Lecture Notes in Mathematics, vol. 676, pp. 1-80 Berlin, Heidelberg, New York: Springer 1978

16. Guillemin, V., Sternberg, S.: Geometric asymptotics. Math. Surv. 14, Am. Math. Soc. (1977)

17. Wolf, J. A.: Remark on nilpotent orbits. Proc. Am. Math. Soc. 51, 213-216 (1975)

18. Arnold, V. I.: Mathematical methods of classical mechanics. Graduate Texts in Mathematics 60, Berlin, Heidelberg, New York: Springer 1978

19. Abraham, R., Marsden, J. E.: Foundations of mechanics. Reading, MA: Benjamin Cummings 1978

Communicated by S.-T. Yau

Received October 30, 1984; in revised form August 2, 1985 
